\title{
Intra-subject sample size effects in plantar pressure analyses
}

\author{
Juliet McClymont ${ }^{1}$, Russell Savage ${ }^{1}$, Todd C Pataky ${ }^{2}$, Robin Crompton ${ }^{1}$, James Charles ${ }^{1}$, Karl Bates $^{\text {Corresp. } 1}$ \\ ${ }^{1}$ Department of Musculoskeletal \& Ageing Science, Institute of Life Course \& Medical Sciences, University of Liverpool, Liverpool, United Kingdom \\ 2 \\ Department of Human Health Sciences,, Kyoto University Graduate School of Medicine, Kyoto,, Japan \\ Corresponding Author: Karl Bates \\ Email address: k.t.bates@liverpool.ac.uk
}

Background. Recent work using large datasets (>500 records per subject) has demonstrated seemingly high levels of step-to-step variation in peak plantar pressure within human individuals during walking. One intuitive consequence of this variation is that smaller sample sizes (e.g. 10 steps per subject) may be quantitatively and qualitatively inaccurate and fail to capture the variance in plantar pressure of individuals seen in larger data sets. However, this remains quantitatively unexplored reflecting a lack of detailed investigation of intra-subject sample size effects in plantar pressure analysis.

Methods. Here we explore the sensitivity of various plantar pressure metrics to intra-subject sample size (number of steps per subject) using a random subsampling analysis. We randomly and incrementally subsample large data sets (>500 steps per subject) to compare variability in three metric types at sample size of 5-400 records: (1) overall whole-record mean and maximum pressure; (2) single-pixel values from five locations across the foot; and (3) the sum of pixel-level variability (measured by mean square error, MSE) from the whole plantar surface.

Results. Our results indicates that the central tendency of whole-record mean and maximum pressure within and across subjects show only minor sensitivity to sample size $>200$ steps. However, $<200$ steps, and particularly $<50$ steps, the range of overall mean and maximum pressure values yielded by our subsampling analysis increased considerably resulting in potential qualitative error in analyses of pressure changes with speed within-subjects and in comparisons of relative pressure magnitudes across subjects at a given speed. Our analysis revealed considerable variability in the absolute and relative response of the single pixel centroids of five regions to random subsampling. As the number of steps analysed decreased, the absolute value ranges were highest in the areas of highest pressure (medial forefoot and hallux), while the largest relative changes were seen in areas of lower pressure (the midfoot). Our pixel-level measure of variability by MSE across the whole-foot was highly sensitive to our manipulation of sample size, such that the range in MSE was exponentially larger in smaller subsamples of $n$ pressure records. Random subsampling showed that the range in pixel-level MSE only came within $5 \%$ of the overall sample size in subsamples $>400$. The range in pixel-level MSE at low subsamples $(<50)$ was $25-75 \%$ higher than that of the full datasets of $>500$ pressure records per subject. Overall, therefore, we demonstrate a high probability that the very small sample sizes ( $n<20$ records), which are routinely used in human and animal studies, capture a relatively low proportion of variance evident in larger plantar pressure data set, and thus may not accurately reflect the true population mean. 


\section{Intra-subject sample size effects in plantar pressure analyses}

2

3 J. McClymont $^{1 *}$, R. Savage ${ }^{1}$, T.C. Pataky ${ }^{2}$, R.H. Crompton ${ }^{1}$ J.P. Charles ${ }^{1}$ and K.T. Bates ${ }^{1}$

4

$5{ }^{1}$ Department of Musculoskeletal \& Ageing Science, Institute of Life Course \& Medical Sciences,

6 The William Henry Duncan Building, 6 West Derby Street, L7 8TX, University of Liverpool,

7 Liverpool, UK.

$8{ }^{2}$ Department of Human Health Sciences, Kyoto University Graduate School of Medicine, Kyoto,

9 Japan

10

$11 *$ Corresponding author e-mail: k.t.bates@liverpool.ac.uk

12

13 Key words: Plantar pressure, variability, walking, sample size, biomechanics.

14

15 


\section{ABSTRACT}

\section{Background.}

18 Recent work using large datasets (>500 records per subject) has demonstrated seemingly high

19 levels of step-to-step variation in peak plantar pressure within human individuals during walking.

20 One intuitive consequence of this variation is that smaller sample sizes (e.g. 10 steps per subject)

21 may be quantitatively and qualitatively inaccurate and fail to capture the variance in plantar

22 pressure of individuals seen in larger data sets. However, this remains quantitatively unexplored

23 reflecting a lack of detailed investigation of intra-subject sample size effects in plantar pressure

24 analysis.

Methods.

27 Here we explore the sensitivity of various plantar pressure metrics to intra-subject sample size

28 (number of steps per subject) using a random subsampling analysis. We randomly and

29 incrementally subsample large data sets ( $>500$ steps per subject) to compare variability in three

30 metric types at sample size of 5-400 records: (1) overall whole-record mean and maximum

31 pressure; (2) single-pixel values from five locations across the foot; and (3) the sum of pixel-

32 level variability (measured by mean square error, MSE) from the whole plantar surface.

34 Results.

35 Our results indicates that the central tendency of whole-record mean and maximum pressure

36 within and across subjects show only minor sensitivity to sample size $>200$ steps. However,

$37<200$ steps, and particularly $<50$ steps, the range of overall mean and maximum pressure values

38 yielded by our subsampling analysis increased considerably resulting in potential qualitative 
39 error in analyses of pressure changes with speed within-subjects and in comparisons of relative

40 pressure magnitudes across subjects at a given speed. Our analysis revealed considerable

41 variability in the absolute and relative response of the single pixel centroids of five regions to

42 random subsampling. As the number of steps analysed decreased, the absolute value ranges were

43 highest in the areas of highest pressure (medial forefoot and hallux), while the largest relative

44 changes were seen in areas of lower pressure (the midfoot). Our pixel-level measure of

45 variability by MSE across the whole-foot was highly sensitive to our manipulation of sample

46 size, such that the range in MSE was exponentially larger in smaller subsamples of $n$ pressure

47 records. Random subsampling showed that the range in pixel-level MSE only came within 5\% of

48 the overall sample size in subsamples $>400$. The range in pixel-level MSE at low subsamples

$49(<50)$ was $25-75 \%$ higher than that of the full datasets of $>500$ pressure records per subject.

50 Overall, therefore, we demonstrate a high probability that the very small sample sizes $(n<20$

51 records), which are routinely used in human and animal studies, capture a relatively low

52 proportion of variance evident in larger plantar pressure data set, and thus may not accurately

53 reflect the true population mean. 


\section{INTRODUCTION}

59 The difficulty of directly assessing internal motion and forces in the distal limbs of humans and

60 animals without resorting to invasive approaches (Lundgren et al., 2008), means that external

61 measures of foot mechanics, such as pressure records, are currently crucial to our understanding

62 of foot function (e.g. Fryberg et al., 1998; Pataky et al., 2008; D'Aout et al., 2009; Crompton et

63 al., 2011; Bates et al., 2013a; DeSilva and Gill 2013). Pressure records are used to diagnose and

64 evaluate abnormalities in the feet and lower limbs of humans (e.g. Fryberg et al., 1998; Pahm et

65 al., 2000; Boulton et al., 2004) and companion animals (e.g. Stadig et al., 2016; Romans et al.,

66 2004), to identify therapeutic interventions (e.g. Arts and Bus 2004; Bus et al., 2008; Paton et

67 al., 2011; Melia et al. 2020) and in furthering our understanding of fall avoidance in the elderly

68 (Xi et al. 2020). They provide key insights into the modulation of foot function (e.g. Simpson et

69 al., 1993; Maluf et al., 2003; Pataky et al., 2008; Stepháne 2008; Caravaggi et al., 2016; Tas

70 and Çetin 2019), in addition to delivering fundamental insights into the evolution of hominid

71 foot morphology and function (e.g. Vereecke et al., 2003; Crompton et al., 2011; Bates et al.,

72 2013a, b; DeSilva and Gill 2013; McClymont and Crompton 2021). Recent work using large

73 intra-subject human datasets ( $>500$ steps per subject) identified high levels of both inter- (i.e.

74 between individuals) and intra-subject (i.e. within subjects step-to-step) variance in peak plantar

75 pressure in the midfoot (Bates et al., 2013a), and across the whole plantar surface in healthy

76 adults (McClymont et al., 2016). This is qualitatively consistent with earlier work measuring

77 variability in loading patterns of neuropathic patients from sample sizes of $>50$ pressure records

78 (Cavanagh et al., 1998). Qualitatively, these findings would imply that a large number of steps

79 might be required to represent the variability and central tendency of an individual's plantar

80 pressure characteristics with a high degree of accuracy. 
81

82 Previous studies that assess intra-subject variability in gait kinematics suggest multiple steps

83 should be analysed to with the aim of capturing the variability of a participant's gait patterns

84 (Cavanagh et al., 1998; Dingwell et al., 2001; Owings \& Grabiner, 2003; Hausdorff et al.,

85 2007; Jordan et al., 2007; Brujin et al., 2013; Riva et al. 2014). For example, Owings \&

86 Grabiner (2003) established 400 steps per subject as a necessary minimum to reliably

87 characterise the motor patterns determining stride length and stride width of an individual

88 participant. While kinematic and spatiotemporal gait parameters have been extensively studied,

89 there has been relatively little research on intra-subject sample size effects in analyses of plantar

90 pressure. The 'one-step', 'two-step', and 'three-step' protocols (van der Leeden et al. 2004) and,

91 the 'five-step mid-gait' approach (McPoil et al., 1999), are comparable as protocols (Oladeji et

92 al. 2008) but are designed to limit small intra-subject sample sizes, to characteristically $n<10$

93 steps per subject (e.g. Taylor et al., 2004). Such protocols are necessary for patients with a low

94 capacity for plantar loading (e.g., patients with diabetic neuropathy or plantar ulcers), but they

95 are still employed in research testing protocols for healthy adults (Taylor et al., 2004; Bus and de

96 Lange 2005; Phethean et al., 2014; McKay et al., 2017; Sole et al., 2017), and children (Hennig

97 et al. 1994; Bosch et al. 2010; Phethean and Nester 2012). Arts \& Bus (2011) conducted a

98 clinical study on patients with diabetic neuropathy assessing variability in numerous plantar

99 pressure variables using an in-shoe Pedar system, orthopedic shoes and non-consecutive steps.

100 Collecting a total sample of 20 non-consecutive steps per foot, the authors concluded that 12

101 mid-gait steps per foot for reliably capturing in-shoe peak pressure patterns (Arts \& Bus, 2011).

102 Kernozek et al., (1996) suggested that 8 steps per foot were required for reliable in-shoe plantar

103 pressures in healthy participants during steady-state treadmill walking. Kiejers et al., (2009)

Peer] reviewing PDF | (2020:12:55991:1:0:NEW 13 May 2021) 
104 collected 17 steps per subject (using young, healthy individuals and the three-step protocol) and

105 suggested that, on average, 3.8 steps per subject were required to meet acceptable levels of

106 reliability in their study context. Where larger sample sizes ( $n=200$ steps $)$ have been considered,

107 only one pressure metric (maximum peak pressure) was evaluated for sensitivity to the number

108 of steps used to quantify the central tendency of individual participants (Melvin et al. 2014).

109 Concerns about sample size have been highlighted by McClymont and Crompton (2021) in the

110 study of fossil footprints (a parallel field to pressure analysis; e.g. Crompton et al. 2011; Bates et

111 al. 2013b), in particular in cases where locomotor behaviour and function are interpreted from a

112 very small sample size of sequential fossil footprints (e.g. Hatala et al. 2016), as usually is the

113 case in this field. Therefore, it is currently unknown how the observed variance in different

114 plantar pressure metrics reported from small sample sizes of steps $(n<50)$, compares to that

115 reported from large sample sizes $(n>500)$ using different pressure metrics in barefoot treadmill

116 walking.

117

118 That intra-subject sample size effects have not been extensively studied despite this breadth of

119 application is perhaps not surprising, given that many experiments pose unique and inherent

120 challenges that may restrict sample size, and influence the characterisation of plantar pressure.

121 For example, in non-companion animal research, intra-subject sample size is limited by access to

122 animals and their willingness to partake in, and follow experimental protocols, limitations that

123 are also commonplace in human paediatric research (Price et al. 2018). In clinical and veterinary

124 contexts, the number of steps per subject is often restricted by physical limitations and efficacy

125 levels that are unique to each patient. As with all kinetic and kinematic gait parameters, valid

126 interpretations of pressure records rests on the assumption that either a single step, or sample of 
127 steps, reliably characterises the foot mechanics of the individual, or population under study, to a

128 degree satisfactory for each analysis. A clear picture of the variance of each subject should be

129 delineated for robust interpretations to be made.

131 The aim of this study is to explore the sensitivity of a number of plantar pressure metrics to intra-

132 subject sample size (number of steps per subject) using a random subsampling analysis. Our goal

133 is to generate insight into sample size-related variability and thereby provide guidance to the

134 design of future plantar pressure analyses.

\section{MATERIALS \& METHODS}

\section{Data Collection}

138 We used an existing, freely available human plantar pressure dataset (McClymont et al., 2016;

139 doi: 10.5061/dryad.09q2b), because of the size and range of walking speeds it encompassed (i.e.

$140>475$ pressure records per subject at $1.1 \mathrm{~m} / \mathrm{s}$ and, $>500$ pressure records per subject per speed at

141 1.3-1.9m/s). According to McClymont et al., (2016) sixteen healthy participants (aged 21-46)

142 with no known or prior limb abnormalities walked barefoot continuously for five minutes, at five

143 different speeds $(1.1 \mathrm{~m} / \mathrm{s}, 1.3 \mathrm{~m} / \mathrm{s}, 1.5 \mathrm{~m} / \mathrm{s}, 1.7 \mathrm{~m} / \mathrm{s}$ and $1.9 \mathrm{~m} / \mathrm{s})$ in random order, on a Zebris FDM-

144 THM pressure sensing treadmill. Anthropometric properties of the dataset are described in

145 McClymont et al., (2016).

147 Data processing

148 Within-footfall maximum pressure values were extracted to yield between 2780-3535

149 pedobarographic records (pressure records; 1 per step) per subject in total from the five speed 
150 trials. Participants with shorter leg lengths took more steps in 5 minutes, producing more

151 pressure records per speed trial, than those with longer leg lengths (Tables S1-2). For each

152 subject, all pressure records from each speed trial were extracted, stacked and registered to each

153 other, using an algorithm that minimises the mean squared error (MSE) between individual

154 pressure records in the stack, and between pixels so that homologous structures optimally

155 overlapped (Pataky \& Goulermas, 2008). Pressure records that varied in length by more than

$1561.5 x$ standard deviations of the mean length or width from each trial were automatically removed

157 from the data set during the registration process to remove anomalous footfalls and footfalls

158 where a portion of the subject's footfall overlapped the edge of the pressure plate embedded in

159 the treadmill. The five registered datasets per subject could then be used to explore the impact of

160 the intra-subject sample size (the number of steps per subject) on foot pressure metrics using our

161 custom written, pixel-wise software, Pedobarographic Statistical Parametric Mapping (pSPM)

162 (Pataky \& Goulermas, 2008; Pataky et al., 2008), in MATLAB (MathWorks, USA).

163

164 Data analysis

165 Investigation of the relationship between sample size and intra-subject plantar pressure variance

166 first required quantitative pressure metrics, and subsequently an analysis of how each metric

167 varies according to the number of steps included in the analysis. Broadly speaking, there are two

168 branches of analyses available to quantify plantar pressure characteristics; simple metrics, where

169 a small number of global (e.g., the maximum and mean pressure from each steps) or regional

170 metrics (e.g., the pressure from the most central pixel in the 'heel' region, the average pressure

171 from pixels in the 'hallux' region); and, pixel-level, vector analyses (e.g., pSPM; Pataky \&

172 Goulermas, 2008; Pataky et al., 2008) that attempt to quantify pressure characteristics from the 
173 whole plantar surface of the foot without spatial data reduction. Here we attempted to investigate

174 the sensitivity of both these types of approaches to intra-subject sample size (Fig. 1). For simple,

175 singular pressure metrics we carried out two intra-subject sample size analyses based on (1) the

176 mean and maximum pressure in each peak plantar pressure within each subject at each speed

177 (Fig. 1A), and (2) the pressure of a single pixel that approximates the centre of 5 locations found

178 commonly in regional analyses ('heel', 'midfoot', 'distal head of the first metatarsal' [MT1],

179 'distal head of the fifth metatarsal' [MT5], and 'hallux') (Fig. 1B). To examine how the number

180 of steps analysed within a subject may influence whole-foot pressure characteristics, and

181 therefore sample size implications for pixel-level vector analyses of pressure (e.g. Pataky \&

182 Goulermas, 2008; Pataky et al., 2008; Bates et al., 2013a, b), we calculated the global mean

183 square error (MSE) of all pixels in each pressure record following McClymont et al., (2016) (Fig.

184 1C). Specifically, the MSE was calculated over every non-zero pixel in each pressure record

185 within the overall total sample from all five speeds according to equation (1):

186

$$
\mathrm{MSE}=\frac{1}{\mathrm{~N}} \sum_{k}^{N}\left(I_{0 k-} I_{1 k}\right)^{2}
$$

where $N$ is the total number of non-zero pixels in the mean image; $\mathrm{I}_{0}$ is the mean of the subject's overall sample and $\mathrm{I}_{1}$ is an individual pressure record. The MSE of each pixel was then summed to produce a total MSE value for each individual pressure record about the subject's overall mean pressure record (Fig. 1C).

192 To explore the sensitivity of these metrics to the number of steps per subject at different speed,

193 we conducted a random subsampling analysis (Fig. 2). 1000 random subsamples of pressure

194 records were extracted from each subject's overall total speed trial at subsamples of 5, 25, 50, 
$195100,200,300,400$ and 500 steps (i.e. 1000 randomly generated samples with an $n$ steps of 5,

1961000 randomly generated samples with an $n$ steps of 25,1000 randomly generated samples with

197 an $n$ steps of 50 etc). The range in mean maximum pressure (mMAXP), the mean of the mean

198 pressures (mMEANP) and mean MSE from within each 1000 subsample at each $n$ steps then was

199 then calculated with respect to the subject's overall mean pressure record (taken from the total

200 sample size of $>500$ steps), enabling quantitative comparison of the sensitivity of each metric to

201 the manipulation of intra-subject sample size (Fig. 2). We ran these subsampling analyses from

202 each speed defined in the dataset $\left(1.1 \mathrm{~ms}^{1}, 1.3 \mathrm{~ms}^{1}, 1.5 \mathrm{~ms}^{1}, 1.7 \mathrm{~ms}^{1}\right.$ and $\left.1.9 \mathrm{~ms}^{1}\right)$ to test for possible

203 changes in response to intra-subject sample size at different walking speeds. Because results

204 remained consistent across all these speeds, we focus on on the results at $1.3 \mathrm{~m} / \mathrm{s}$ subsequent

205 sections, as it represents a walking speed easily and regularly practiced by healthy young adults

206 in everyday life (Himann et al., 1988; Oberg et al., 1993; Samson et al., 2001). The results from

207 speeds $1.1 \mathrm{~m} / \mathrm{s}, 1.5 \mathrm{~m} / \mathrm{s}, 1.7 \mathrm{~m} / \mathrm{s}$ and $1.9 \mathrm{~m} / \mathrm{s}$, presented separately in the supplementary material 208 (Figs S1-8).

209

210 RESULTS

211 Sensitivity of simple 'whole-foot' pressure metrics to intra-subject sample size

212 Across all speeds, all participants showed a qualitatively similar pattern of response to random

213 subsampling of mMEANP and mMAXP (Fig. 3A). The average range (for the 16 subjects) in the

214 mMAXP values from 1000 randomly subsampled populations at $1.3 \mathrm{~m} / \mathrm{s}$, remained less than

$2152 \mathrm{~N} / \mathrm{cm}^{2}$ for sample sizes of $100-500$ steps per subject but, increased sharply when the number of

216 steps for an individual subject was reduced to 50 or less (Fig. 3A). At 5 steps per subject, the

217 average range in mMAXP values was $7.1 \mathrm{~N} / \mathrm{cm}^{2}$, with the highest and lowest subjects yielding 
218 values of $4.7 \mathrm{~N} / \mathrm{cm}^{2}$ and $10.3 \mathrm{~N} / \mathrm{cm}^{2}$ (Fig. 1A). These values at an $n$ of five steps corresponded to

219 percentage errors of $13.2 \%$ for the average across the subjects, and $8.5 \%$ and $18.2 \%$ for the

220 highest and lowest subjects (Fig. 3C). Similar trends were seen in the range in mMEANP (Figs

221 1B \& D); the average range across subjects remained below $0.1 \mathrm{~N} / \mathrm{cm}^{2}$ for sample sizes of 100 -

222500 steps per subject, but increased sharply when the number of steps was reduced to 50 or less

223 (Fig 3B). At 5 steps per subject, the average range in mMEANP values was above $0.3 \mathrm{~N} / \mathrm{cm}^{2}$,

224 with the highest and lowest subjects yielding values of $0.1 \mathrm{~N} / \mathrm{cm}^{2}$ and $0.5 \mathrm{~N} / \mathrm{cm}^{2}$ (Fig. 3B). A

225 corresponding percentage error of $7.7 \%$ was found for the average across the subjects, with $4.8 \%$

226 and $10.4 \%$ for the highest and lowest subjects (Fig. 3D).

227

228 To place these magnitudes in a comparative context, Figure 4A \& B show how the range of 229 mMEANP and mMAXP values given by the 1000 randomly generated subsamples, changes

230 across 5-500 steps within a single subject at all five walking speeds. Only at sample sizes of 200

231 or more steps were the qualitative difference between speeds (i.e. higher mMEANP and

232 mMAXP values at higher walking speeds) preserved. At 200 or fewer steps for mMAXP and 50

233 or fewer steps for mMEANP (Fig. 4A-B), the range in values produced at adjacent walking

234 speeds began to overlap, raising the possibility of comparative analyses failing to correctly

235 identify the qualitative difference between walking speeds (i.e. falsely suggesting a decrease in

236 mean and maximum pressure with increasing walking speed). Figures 4C-D show the range of

237 mMEANP and mMAXP values given by the 1000 randomly generated subsamples changes

238 across 5-500 steps for five randomly selected subject walking at $1.3 \mathrm{~m} / \mathrm{s}$. For mMAXP, the

239 correct qualitative differences between subjects were preserved until the number of steps was

240 reduced to 25 or less, when the ranges between adjacent subjects overlap, indicating the potential 
241 for an analysis to incorrectly identify relative pressure differences between subjects (Fig. 4C).

242 For mMEANP, the situation is more complex: subject 5 shows very low mMEANP at $1.3 \mathrm{~m} / \mathrm{s}$

243 and their range in pressure magnitudes remained lower than all other subjects regardless of the

244 number of steps used (Fig. 4D). However, the other four randomly selected subjects began to

245 show overlapping ranges in mMEANP at steps of 200 or less, with high levels of overlap (and

246 therefore potential for incorrect identification of relative pressures) at 25 or fewer steps (Fig.

247 4D).

248

249 Sensitivity of single-pixel region centroid values to intra-subject sample size

250 Across all speeds, all participants showed a qualitatively similar pattern of response to random

251 subsampling of mean values for the centroid pixels of the five-foot regions: 'heel', 'midfoot',

252 lateral ('MT5') and medial ('MT1') forefoot, and 'hallux' (Fig. 1B), although the absolute and

253 relative magnitudes of responses varied considerably across regions and between subjects (Figs

254 5-6). The average range (for the 16 subjects) in the mean values given by the 1000 randomly

255 subsampled populations at $1.3 \mathrm{~m} / \mathrm{s}$ remained less than $3 \mathrm{~N} / \mathrm{cm}^{2}$ for sample sizes of $>200$ steps per

256 subject but, increased sharply in all five regions when the number of steps for an individual

257 subject was reduced to 100 or less (Fig. 5). At five steps per subject, the average range in mean

258 values exceeded $8 \mathrm{~N} / \mathrm{cm}^{2}$ in all five regions, with the highest absolute values occurring in the

259 medial forefoot ('MT1') and hallux (Fig. 5D-E) and the lowest absolute values in the 'midfoot'

260 (Fig. 5B). Within each region there was considerable inter-subject variation in absolute range

261 magnitudes versus subsample size. For example, at five steps per subject, the subjects with

262 largest and smallest absolute ranges yielded values of $9.74 \mathrm{~N} / \mathrm{cm}^{2}$ versus $0.97 \mathrm{~N} / \mathrm{cm}^{2}$ in the

263 'midfoot' (Fig. 5B), and $22.12 \mathrm{~N} / \mathrm{cm}^{2}$ versus $5.24 \mathrm{~N} / \mathrm{cm}^{2}$ in the medial ('MT1') forefoot (Fig. 5D) 
264 respectively. When ranges were normalized as percentage of the pixel values at the full sample

265 size, the 'midfoot' centroid pixel shows the highest relative error of all five regions (Fig. 6) with

266 a mean value for the subjects of $171 \%$, and $672 \%$ versus $41.74 \%$ for the subjects with highest

267 and lowest values (Fig. 6B). The lowest percentage values were seen in the 'heel' centroid pixel,

268 where the mean values for the subjects was $12.16 \%$, with the highest and lowest subject values

269 recovered as $22.4 \%$ versus $3.07 \%$ (Fig. 6 A).

270

271 Sensitivity of whole-foot MSE to intra-subject sample size.

272 At $1.3 \mathrm{~m} / \mathrm{s}$, the range of whole-foot mean MSE given by the 1000 randomly generated

273 subsamples reduced exponentially as subsampled number of steps increased (Fig. 7A, Figs S1-8,

274 Tables S1-2). All results for other speeds follow the same exponential curve (see Figs S1-8). The

275 number of pressure records required for the range in mean MSE to be within 5\% of the overall

276 dataset mean MSE range was $n=401$ records at $1.3 \mathrm{~m} / \mathrm{s}$ (Fig. 7B, Tables S1-2). In smaller

277 subsamples (i.e., $n=<10$ ) the range in mean MSE exceeded $75 \%$ of the overall sample range in 278 mean MSE (Fig. 7).

279

280

\section{DISCUSSION AND CONCLUSIONS}

281 The aim of this study was to understand the stability of a number of widely used plantar pressure 282 metrics (Fig. 1) when exposed to random manipulations of sample size (Figs 3-7). To this end, 283 we hoped to provide insight into the effect that intra-subject sample size (the number of steps 284 analysed within a subject) has on the estimative and interpretive power in assessing variance in 285 peak plantar pressure, to provide general guidance to the design of plantar pressure analyses. 
287 The level of accuracy or reliability required for an analysis depends on the goals of the

288 experiment or the specific hypotheses being tested. The effects of the number of steps analysed

289 per subject derived herein provide general guidance in this respect for a number of metrics in

290 healthy human participants during steady-state, barefoot, treadmill walking. During continuous

291 speed trials, the observed variation in each pressure record is not entirely independent from each

292 other, rather each step is part of a feedback loop in the motor control of walking, whereby a

293 walking pattern in any gait cycle may influence subsequent strides in accordance with fractal

294 dynamics (Terrier et al., 2011). Thus, the continuous collection of large numbers of pressure

295 records reduces the risk of observing higher variability than would be reported from smaller

296 sample sizes. Higher variability will likely be observed from short recording sessions with a low

297 intra-trial $n$. However an analysis of this effect from non-continuously collected pressure records 298 remains to be performed.

300 In this study we chose to compare variability in a number of different pressure metrics (Fig. 1)

301 across incrementally subsampled numbers of steps per subjects collected during five minutes of

302 continuous walking at a range of walking speeds $(1.1-1.9 \mathrm{~m} / \mathrm{s})$. However, we recognise that our

303 experiment does not reflect many other circumstances in biomechanics research where plantar

304 pressure is analysed. In some human studies (e.g. Kernozek et al., 1996; Cavanagh et al., 1998;

305 Frykberg et al., 1998; Pham et al., 2000; Boulton et al., 2004; Bus et al., 2008; Paton et al.,

306 2011; Arts \& Bus, 2011) and in animal research (Romans et al., 2004; Michilsens et al., 2009;

307 Stadig et al., 2016; Panagiotopoulou et al., 2016), plantar pressure is typically recorded during

308 unrestricted over-land locomotion using free-standing pressure mats or plates. In these

309 circumstances the environmental conditions that the participant encounters inherently differs 
310 from treadmill locomotion, and furthermore non-sequential steps are common. Caution should

311 therefore be taken in applying our treadmill results to overground datasets until further tests can

312 confirm the effects. However, we would predict broadly similar exponential increases in variance

313 with a small number of steps if our subsampling protocol were applied to such data sets.

314

315 Our analysis of simple 'whole-foot' pressure metrics (Figs 1A, 3-4) demonstrates a complex

316 interaction between the number of steps used per subject and the specific pressure metric chosen

317 (mMEANP versus mMAXP) to represent a step with a single aggregate value. In general, our

318 analysis suggests that at 200 steps or more, the absolute and relative 'error' in both mMEANP

319 and mMAXP relative to a sample of 500-600 records is likely to be very low (Figs 3-4). As a

320 result, 200-500 records (in our analysis) yield the same qualitative patterns or results, both in the

321 intra-subject analysis of pressure magnitude for walking speed (Figs 4A-B), and, in a cross-

322 subject comparison at $1.3 \mathrm{~m} / \mathrm{s}$ (Figs 4C-D). At fewer than 200 steps (and particularly at $<50$

323 steps) the absolute and relative 'error' in simple pressure metrics relative to a sample of 500-600

324 records, begins to increase approximately exponentially (Figs 3-4). mMAXP appears to yield

325 greater error magnitudes than mMEANP (Figs 3C-D), yet in our comparative analysis,

326 mMEANP was clearly more susceptible to qualitative error in terms of the potential to

327 incorrectly represent relative differences in pressure across subjects at lower samples sizes (Fig. 328 4D).

330 The mean value of single pixels at the centroid of our five regions (Fig. 1B) vary considerably in

331 their absolute (Fig. 5) and relative (Fig. 6) response to subsampling. Perhaps not surprisingly,

332 higher pressure regions, particularly the medial midfoot ('MT1') and hallux, are recovered with 
333 the highest absolute ranges as increasingly fewer steps are used to derive the mean pressure (Fig.

334 5D-E). This finding is consistent with the MSE 'variation maps' (Fig. 1C) for this same subject

335 data set presented by McClymont et al., (2016), which demonstrated that average MSE was

336 broadly correlated with pressure magnitudes across the foot and therefore highest in the medial

337 forefoot and hallux. McClymont et al., (2016) also examined the spatial variation in co-efficient

338 of variation $(\mathrm{CoV})$ of peak pressure across the whole plantar surface of the foot. These $\mathrm{CoV}$

339 variation maps are consistent our relative assessment of intra-subject sample size effects in

340 different foot regions (Fig. 6), which is perhaps not surprising given that both are forms of

341 normalised measurements. Normalised assessments suggest that step-to-step variability

342 (McClymont et al., 2016) and subsequently sensitivity to sample size ( $n$ steps) is relatively higher

343 in lower pressure regions of the foot, and particularly the midfoot (Fig. 6B). As a result, many

344 subjects show a range in mean values in excess of $25 \%$ of the full data set mean for the midfoot

345 at less than 300 steps, and on average, our subjects yield a range of mean values above $100 \%$ of

346 the full data set mean when less than 15 steps were used (Fig. 6B). This contrasts considerably

347 with higher pressure regions in the heel and forefoot where the mean range for all subjects only

348 reaches $10-30 \%$ the value recovered using all steps ( $\mathrm{n}>500$ steps) when less than 10 steps are

349 used to calculate the mean (Fig. 6).

350

351 The range of whole-foot mean MSE at low sample sizes $(n<10)$ is $75 \%$ higher than the overall

352 dataset mean MSE range of $<500$ (Fig. 7). Furthermore, the magnitude of the sensitivity of the

353 mean MSE to subsample $n$, is highly variable between individuals (Fig. 7). For example, at a

354 walking speed of $1.3 \mathrm{~m} / \mathrm{s}$ and a subsample $n=10$, subject 7 (highest MSE) and subject 13 (lowest

355 MSE), had a MSE range of 10.3 and 2.2 respectively. However, at $n=400$, their MSEs were 
356 reduced to 1.5 and 0.4 respectively (Fig. 7) and to within 5\% of the overall dataset mean for

$3571.3 \mathrm{~m} / \mathrm{s}$. This result suggests that many pressure records $(n>400)$ are required to reflect the high

358 levels of observed variation in peak pressure given by the full dataset (Fig. 7). This suggests that

359 at small sample sizes $(n=<25)$, there is a high probability that neither the range of variation nor

360 the mean peak pressure will be reflected as accurately as it would if collected from larger

361 datasets $(n>400)$ (Fig. 7).

362

363 Our finding that a relatively high number of steps are required to capture a high proportion of the

364 variability in different pressure metrics present in the full datasets (e.g. $n>100$ steps in

365 mMEANP and mMAXP, Figs 3-4; $n>300$ steps for single 'midfoot' pixel values, Fig. 6E; $n$

$366>400$ steps in whole-foot MSE values, Fig. 7), compliments other reliability studies that assess

367 variability in kinematic data (e.g. Owings \& Grabiner, 2003; Bruijin et al., 2013). However,

368 these results are not entirely consistent, at least qualitatively, with previous reliability studies

369 specific to plantar pressure (e.g. Kernozek et al., 1996; Keijsers et al., 2009; Arts \& Bus, 2011),

370 and suggest that a considerably larger $n$ is necessary to capture pressure patterns. As mentioned

371 previously, earlier clinical assessments of variability in plantar pressure concluded that between

372 4-12 pressure records to be a valid and reliable interpretation of peak plantar pressure in patients

373 with diabetic neuropathy (Kernozek et al., 1996; Keijsers et al., 2009; Arts and Bus., 2011). As a

374 general guide to capturing pressure characteristics, these small step numbers are lower than our

375 results from the simple pressure metrics suggest are appropriate in healthy subjects (Figs 3-4).

376 Our results are more similar to those of Melvin et al. (2014) who suggested that a minimum of

37730 steps per foot should be collected to accurately represent a subject's mMAXP. Variation

378 across studies in their goals or hypotheses, experimental protocols (see discussion above), 
379 equipment, subject characteristics and health status, and in the analytical methods used all

380 combine to make it difficult to assess why our findings suggest, at least qualitatively, that a

381 relatively large number of steps are required to accurately represent the central tendency of an

382 individual's peak pressure magnitude and distribution (Figs 3-7) compared to previous work

383 Kernozek et al., 1996; Keijsers et al., 2009; Arts and Bus., 2011).

384

385 Furthermore, our study perhaps considers 'reliability' in a different context to most, if not all,

386 previous studies. Previous studies explicitly or implicitly view reliability in its classical sense: as

387 the ratio of between- vs. within-subject variability. When this ratio is high, the data are generally

388 considered to be 'reliable'. From this perspective, a relatively small sample size (e.g. $n=10$ steps

389 or less) is sufficient for generating reliable conclusions because between-subject variability is

390 usually very large compared to trial-to-trial or step-to-step variability. In contrast to this classical

391 perspective, our paper considered 'reliability' in terms of longer-term within-subject behaviour,

392 which we defined in terms of metric value convergence over a large number of trials. Although

393 this perspective is defined in terms of within-subject 'reliability', it also has important

394 implications for between-subjects analysis. For example, the potential for miscalculating or

395 misinterpreting pressure patterns because of small sample sizes can be further illustrated by

396 visualizing the range in peak pressure in a small number of peak pressure records (Fig. 8). Figure

3978 shows the first 12 steps at $1.3 \mathrm{~m} / \mathrm{s}$ produced by the participants with the highest (subject 7) and

398 lowest (subject 13) mean MSE in our pixel-level analysis (Fig. 7). The overall pressure

399 distribution and the position of peak pressure shifts over the plantar surface in both participants

400 step-to-step, and very few of these records qualitatively resemble the mean pressure image (Fig.

401 8). Furthermore, participants in this study did not show the large variance in midfoot pressure 
402 noted in other healthy participants in a previous study (Bates et al., 2013a) where midfoot

403 pressure varied step-to-step from minimum to maximum pressure across the plantar surface in

404 some individuals. Presumably, participants exhibiting such extreme ranges in mid-foot pressure

405 would show even more variation over the plantar surface than those analysed herein.

407 Technological advances in data collection (e.g. instrumented treadmills) and analysis (e.g.

408 automated image analysis algorithms and pixel-level statistical comparisons) are making

409 interrogation of very large biomechanical datasets increasingly feasible. These advances are

410 important given the revelation of seemingly high levels of intra-subject or step-to-step variance

411 in pressure magnitude and distribution in recent analyses of larger than average pressure datasets

412 (Bates et al., 2013a; McClymont et al., 2016). Our results demonstrate that at the largest sample

413 sizes typically collected in plantar pressure analyses $(n=20-50$ records) the range of mean MSE

414 values given by the 1000 randomly generated subsamples is more than $50 \%$ higher than the

415 mean MSE of the full dataset of $<500$ (Fig. 7). At $n<10$ records, this increases to more than

$41675 \%$, indicating a high probability that such a sample of records will not reflect either the range

417 of variance or the mean pressure given by a larger dataset of $n=500$ (Fig. 7). Sample-size

418 related reliability is specific to experimental goals and hypotheses thus caution must be taken

419 when evaluating individual studies in the context of these results. However, the potential

420 implications of these results (Figs 3-8) are clearly non-trivial, given that most hypotheses

421 regarding human foot development, change and function, are derived from sample sizes of 3-30

422 pressure records per subject (Hughes et al., 1991; Hennig and Rosenbaum 1994; Cavanagh et

423 al., 1997; Bernfield et al., 2004; Segal et al., 2004; Warren et al., 2004; Yang et al., 2005; 
424 Drerup et al., 2008), with only three examples of single participant trials comprising $n=50$

425 records (Rosenbaum et al., 1994; Taylor et al. 2004; Melvin et al., 2014).

426

427 ACKNOWLEDGEMENTS

428 This work is dedicated to the colourful life of Russell Savage, who sadly passed away before this 429 work was published. Without his dedication and scientific creativity, this work would not have 430 been possible. We also thank the many volunteer participants for giving up their time to help 431 create such a large dataset. Thanks to Dr. Steven Lang for guidance on statistical terminology for 432 reliability thresholds.

434 FUNDING STATEMENT

435 This work was funded by the Institute of Ageing and Chronic Disease, University of Liverpool, a 436 National Environment Research Council grant (NE/H004246/1) to R.H.C., and a Leverhulme 437 Trust grant (RPG-2017-296) to K.T.B.

438

439

440

441 REFERENCES

442 Arts MLJ, Bus SA. 2011. Twelve steps per foot are recommended for valid and reliable in-shoe

443 plantar pressure data in neuropathic diabetic patients wearing custom made footwear. Clin.

444 Biomech. 26:880-884. 
445 Bates KT, Collins D, Savage R, Webster E, Pataky TC, McClymont J, D’Aout K, Sellers

446 WI, Bennett MR, Crompton RH. 2013a. The evolution of compliance in the human lateral

447 mid-foot. Proceedings of Royal Society B 280: 20131818

448 Bates KT, Savage R, Pataky TC, Morse SA, Webster E, Falkingham PL, Ren L, Qian Z,

449 Collins D, Bennett MR, McClymont J, Crompton RH. 2013b. Does footprint depth correlate

450 with foot motion and pressure? J. R. Soc. Interface 10:20130009.

451 Bernstein NA. 1967. The coordination and regulation of movements. Ed. Bernstein N.A:

452 Pergamon Press.

453 Boulton AJ, Kirsner RS, Vileikyte L. 2004. Clinical practice. Neuropathic diabetic foot ulcers.

454 New Engl. J. Med. 351:48-55.

455 Bruijn S, Meijer O, Beek P., van Dieën, J. 2013. Assessing the stability of human locomotion:

456 A review of current measures. J. R. Soc. Interface 83:20120999.

457 Burnfield JM, Few CD, Mohamed OS, Perry J. 2004. The influence of walking speed and

458 footwear on plantar pressure in older adults. Clin. Biomech. 19:78-84.

Bus, SA, Valk GD, van Deursen RW, Armstrong DG, Caravaggi C, Hlavacek P. et al. 2008.

460 The effectiveness of footwear and offloading interventions to prevent and heal foot ulcers and

461 reduce plantar pressure in diabetes: a systematic review. Diabetes-Metab. Res. 24 (Supp.

462 1):S162-S180.

463 Caravaggi P, Leardini A, Giacomozzi C. 2016. Multiple linear regression approach for the

464 analysis of the relationships between joints mobility and regional pressure-based parameters in

465 the normal-arched foot. Journal of Biomechanics 49:3485-3491.

466 Cavanagh PR, Morag E, Boulton AJ, Young MJ, Deffner KT, Pammer SE. 1997. The

467 relationship of static foot structure to dynamic foot function. J. Biomech 30:243-250. 
468 Cavanagh P, Perry JE, Ulbrecht JS, Derr JA, Pammer SE. 1998. Neuropathic diabetic

469 patients do not have reduced variability of plantar loading during gait. Gait Posture 7:191-199.

470 Crompton RH, Pataky TC, Savage R, D'Aout K, Bennett MR, Day MH, Bates KT, Morse S,

471 Sellers WI. 2012. Human-like external function of the foot, and fully upright gait, confirmed in

472 the 3.66 million year old Laetoli hominin footprints by topographic statistics, experimental

473 footprint-formation and computer simulation. Journal of the Royal Society Interface 9:707-719.

474 D'Août K, Pataky T, De Clercq D, Aerts P. 2009. The effects of habitual footwear use: foot

475 shape and function in native barefoot walkers. Footwear Science 1:81-94.

476 Dingwell J, Cusumano J, Cavanagh P, Sternad D. 2001. Local dynamic stability versus

477 kinematic variability of continuous overground and treadmill walking. J. Biomech. Eng. 123: 2747832.

479 Drerup B, Szczepaniak A, Wetz HH. 2008. Plantar pressure reduction in step-to gait: a

480 biomechanical investigation and clinical feasibility study. Clinical Biomechanics 23:1073-1079.

481 Frykberg RG, Lavery LA, Pham H, Harvey C, Harkless L, Veves, A. 1998. Role of

482 neuropathy and high foot pressures in diabetic foot ulceration. Diabetes Care 21:1714-1719.

483 Georgia M, Siegkas P, Levick J, Apps C. 2020. Insoles of uniform softer material reduced

484 plantar pressure compared to dual-material insoles during regular and loaded gait. Applied

485 Ergonomics 91:103298.

486 Glazier PS, Davids K. 2009. Constraints on the Complete Optimization of Human Motion.

487 Sports Med. 39:15-28.

488 Hatala KG, Roach NT, Ostrofsky KR, Wunderlich RE, Dingwall HL, Villmoare BA, Green

489 DJ, Harris JWK, Braun DR, Richmond BG. 2016. Footprints reveal direct evidence of group

490 behaviour and locomotion in Homo erectus. Scientific reports 6:28766. 
491 Hausdorff JM. 2007. Gait dynamics, fractals and falls: finding meaning in the stride to stride

492 fluctuations in human walking. Hum. Movement Sci. 26:555-589.

493 Jordan K, Challis JH, Newell KM. 2007. Walking speed influences on gait cycle variability.

494 Gait Posture 26:128-134.

495 Hennig D, Rosenbaum EM. 1991. Pressure distribution patterns under the feet of children in

496 comparison with adults. Foot Ankle Int. 11:306-311.

497 Himann JE, Cunningham DA, Rechnitzer PA, Paterson DH. 1988. Age-related changes in 498 speed of walking. Med. Sci. Sports. Exerc. 20:161-166.

499 Hughes J, Pratt L, Linge K, Clark K, Klenerman L. 1991. Reliability of pressure

500 measurements: the EMED F system. Clin. Biomech. 6:14-18.

501 Kernozek TW, LaMott EE, Dancisak MJ. 1996. Reliability of an in-shoe pressure

502 measurement system during treadmill walking. Foot Ankle Int. 17:204-209.

503 Latash M. 2000. There is no motor redundancy in human movements. There is motor

504 abundance. Motor Control. 4:259-261.

505 Latash ML, Scholz JF, Schöner FDG. 2001. Structure of motor variability in marginally

506 redundant multi-finger force production tasks. Exp. Brain. Res. 141:153-165.

507 Lundgren P, Nester C, Arndt A, Jones R, Stacoff A, Wolf P, Lundberg A. 2008. Invasive in 508 vivo measurement of rear-, mid- and forefoot motion during walking. Gait Posture 28:93-100.

509 McPoil TG, Cornwall MW, Dupuis L, Cornwell M. 1999. Variability of plantar pressure data.

510 A comparison of the two-step and midgait methods. Journal of the American Podiatric Medical 511 Association 89:495-501. 
512 Maluf KS, Mueller MJ. 2003. Comparison of physical activity and cumulative plantar tissue

513 stress among subjects with and without diabetes mellitus and a history of recurrent plantar

514 ulcers. Clin. Biomech.18:567-575.

515 McClymont J, Pataky TC, Savage R, Crompton RH, Bates KT. 2016. Functional variability

516 in plantar pressure during a range of controlled walking speeds. Roy. Soc. Open Sci. 3:160369.

517 McClymont J, Crompton RH. 2021. 'Repetition without Repetition': a comparison of the

518 Laetoli G-1, Ileret, Namibian Holocene and Modern Human footprints using pedobarographic

519 Statistical Parametric Mapping. In: Pastoors, A. and Tlensen-Erz, T, eds. Reading Prehistoric

520 Human Tracks Berlin: Springer.

521 McKay MJ, Baldwin JN, Ferreira P, Simic M, Vanicek N, Wojciechowski E, Mudge A,

522 Burns J. and 1000 Norms Project Consortium. 2017. Spatiotemporal and plantar pressure

523 patterns of 1000 healthy individuals aged 3-101 years. Gait \& posture 58:78-87.

524 Melvin JMA, Preece S, Nester CJ, Howard D. 2014. An investigation into plantar pressure

525 measurement protocols for footwear research. Gait \& Posture 40:682-687.

526 Michilsens F, Aerts P, van Damme R, D'Aout K. 2009. Scaling of plantar pressures in

527 mammals. J. Zoology 279:236-242.

528 Newell KM, Corcos DM. 1993. Variability and Motor Control in Issues in variability and motor

529 control. In: eds. Newell ,K.M., Corcos, D.M., Champaign, Human Kinetics.

530 Oberg T, Karsznia A, Oberg K. 1993. Basic gait parameters: reference data for normal

531 subjects, 10-79 years of age. J. Rehab. Res. Devel. 30:210.

532 Oladeji O, Stackhouse C, Gracely E, Orlin M. 2008. Comparison of the two-step and midgait

533 methods of plantar pressure measurement in children. J. Am. Podiat. Med. Assoc. 98:268-77. 
534 Owings TM, Grabiner MD. 2003. Measuring step kinematic variability on an instrumented

535 treadmill: how many steps are enough? J. Biomech. 36:1215-1218.

536 Panagiotopoulou O, Pataky PC, Day M, Hensman MC, Hensmen S, Hutchinson JR. 2016.

537 Foot pressure distributions during walking in African elephants (Loxodonta africana). R. Soc.

538 Open Sci. 3:160203.

539 Pataky TC, Goulermas JY. 2008. Pedobaragraphic statistical parametric mapping (pSPM): A

540 pixel-level approach to plantar pressures image analysis. J. Biomech. 41:2136-2143.

541 Pataky TC, Caravaggi P, Savage R, Parker, D, Goulermas JY, Sellers WI, Crompton RH.

542 2008. New insights into the plantar pressure correlates of walking speed using pedobarographic

543 statistical parametric mapping (pSPM). J. Biomech. 41:1987-1994.

544 Paton J, Bruce G, Jones R, Stenhouse E. 2011. Effectiveness of insoles used for the prevention

545 of ulceration in the neuropathic diabetic foot: a systematic review. J. Diabetes Complicat. 25:5254662.

547 Pham H, Armstrong DG, Harvey C, Harkless LB, Giurini JM, Veves A. 2000. Screening

548 techniques to identify people at high risk for diabetic foot ulceration: A prospective multicenter

549 trial. Diabetes Care 23:606-611.

550 Phethean J, Nester C. 2012. The influence of body weight, body mass index and gender on

551 plantar pressures: results of a cross-sectional study of healthy children's feet. Gait Posture

552 36:287-90.

553 Phethean J, Pataky TC, Nester CJ, Findlow AH. 2014. A cross-sectional study of age-related

554 changes in plantar pressure distribution between 4 and 7 years: a comparison of regional and 555 pixel-level analyses. Gait Posture 39:154-60. 
556 Price C, McClymont J, Hashmi F, Morrison SC, Nester C. 2018. Development of the infant

557 foot as a load bearing structure: study protocol for a longitudinal evaluation (the Small Steps

558 study). Journal of Foot and Ankle Research 11:33.

559 Riva F, Bisi MC, Stagni R. 2014. Gait variability and stability measures: Minimum number of

560 strides and within-session reliability. Computers in Biology and Medicine 50:9-13.

561 Romans CW, Conzemius MG, Horstman CL, Gordon WJ, Evans RB. 2004. Use of pressure

562 platform gait analysis in cats with and without bilateral onychectomy. A. J. Vet. Res. 65:1276-

5631278.

564 Rosenbaum D, Hautmann S, Gold M, Claes L. 1994. Effects of walking speed on plantar

565 pressure patterns and hindfoot angular motion. Gait Posture 2:191-197.

566 Samson MM, Crowe A, de Vreede PL, Dessens JAG, Duursma SA, Verhaar HJJ. 2001.

567 Differences in gait parameters at a preferred walking speed in healthy subjects due to age, height

568 and body weight. Ageing Clin. Ex. Res. 13:16-21.

569 Schaller NU, D’Aout K, Villa R, Herkner B, Aerts P. 2011. Toe function and dynamic

570 pressure distribution in ostrich locomotion. J. Ex. Bio. 214:1123-1130.

571 Segal A, Rohr E, Orendurff M, Shofer J, O'Brien M, Sangeorzan B. 2004. The effect of

572 walking speed on peak plantar pressure. Foot Ankle Int. 25:926-933.

573 Simpson KJ, Jiang P, Shewokis PA, Odum S, Reeves KT.1993. Kinematic and plantar

574 pressure adjustments to downhill gradients during gait. Gait Posture 13:172-179.

575 Stadig SB, Lascelles DX, Bergh A. 2016. Do cats with a cranial cruciate ligament injury and

576 osteoarthritis demonstrate a different gait pattern and behaviour compared to sound cats? Acta.

577 Vet. Scan. 58:1-70.

Peer) reviewing PDF | (2020:12:55991:1:0:NEW 13 May 2021) 
578 Stéphane S, Vereecke E, Rooze M, Van Sint Jan S. 2008. Functional Anatomy of the Foot.

579 Part A: The Modern Human Foot. In: K. D'Août, B. Lescrenier, B. Van Gheluwe \& D. Damien

580 De Clercq, eds. Advances in Plantar Pressure Measurements in Clinical and Scientific

581 Research. Maastricht: Shaker Publishing, pp. 69-91.

582 Sole G, Pataky T, Sole CC, Hale L, Milosavljevic S. 2017. Age-related plantar centre of

583 pressure trajectory changes during barefoot walking. Gait \& Posture 57:188-192.

584 Taş S, Çetin A. 2019. An investigation of the relationship between plantar pressure distribution

585 and the morphologic and mechanic properties of the intrinsic foot muscles and plantar

586 fascia. Gait \& Posture 72:217-221.

587 Taylor AJ, Menz HB, Keenan AM. 2004. The influence of walking speed on plantar pressure

588 measurements using the two-step gait initiation protocol. The Foot 14:49-55.

589 Terrier P, Olivier D. 2011. Kinematic variability, fractal dynamics and local dynamic stability

590 of treadmill walking. J Neuro. Eng. Rehab. 8:1-13.

591 van der Leeden M, Dekker JH, Siemonsma PC, Lek-Westerhof SS, Steultjens MP. 2004.

592 Reproducibility of plantar pressure measurements in patients with chronic arthritis: a comparison

593 of one-step, two-step, and three-step protocols and an estimate of the number of measurements

594 required. Foot \& Ankle International 25:739-744.

595 Vereecke E, D’Aout K, Clercq DD, Van Elsacker L, Aerts P. 2003. Dynamic plantar pressure

596 distribution during terrestrial locomotion in bonobos (Pan paniscus). Am. J. Phys. Anthropol.

597 120:373-383.

598 Warren GL, Maher RM, Higbie EJ. 2004. Temporal patterns of plantar pressure and lower-leg

599 muscle activity during walking: effect of speed. Gait Posture 19:91-100. 
600 Xi X, Jiang W, Lü Z, Miran SM, Luo ZZ. 2020. Daily activity monitoring and fall detection

601 based on surface electromyography and plantar pressure. Complexity 2020:9532067.

602 Yang S, Chiu YI, Lin CC, Chen YH. 2005. Influence of walking speed to the peak plantar

603 pressure response. In: Seventh Symposium on Footwear Biomechanics, Cleveland, USA.

604 Zhu H, Wertsch JJ, Harris GF, Alba HM. 1995. Walking cadence effect on plantar pressures. 605 Arc.h Phys. Med. Rehab. 76:1000-1005.

\section{FIGURE CAPTIONS}

607 Figure 1. Overview of the plantar pressure metrics used in the intra-subject (number of steps)

608 subsampling analysis. We investigated the sensitivity of three different metric types to the

609 number of steps analysed within each subject. These were (A) the simple 'whole-foot' mean

610 (mMEANP) and maximum pressure (mMAXP), and (B) the absolute values of pixels

611 representing the approximate centroid of the heel, midfoot, lateral and medial forefoot (MT5 and

612 MT1 above) and the hallux. (C) To examine how the number of steps analysed within a subject

613 may influence the foot as a whole (i.e. the sum of variability across all pixels in each record), and

614 therefore sample size implications for pixel-level vector analyses of pressure we calculated the

615 global mean square error (MSE) of all pixels in each pressure record following McClymont et al., 616 (2016).

617

618 Figure 2. Conceptual example of the subsampling analysis applied to the various pressure

619 metrics shown in Figure 1. (A) 1000 random subsamples of the pressure metrics were extracted

620 from each subject's overall total number of steps at subsamples of 5, 25, 50, 100, 200, 300, 400

621 and 500 steps (represented as blue diamond data points in A). As the number of steps included in 
622 any one of 1000 subsampled populations decreases it would be expected that the range in the

623 absolute value of the metric pressure metric would increase, reflecting (for example) the

624 relatively greater impact of 'extreme' high and low values from specific steps. In other words,

625 the absolute range of values for the pressure metric (dashed blue lines delimiting the maximum -

626 minimum values from the 1000 subsampled populations) would be expected to increase as the

627 number of steps included decreases, as shown in (B). The absolute and relative magnitude of

628 metric ranges are plotted against sample size (n steps) in subsequent plots to make comparisons

629 across subjects and speeds, as shown in (B).

630

631 Figure 3. The relationship between intra-subject sample size (number of steps per subject) and

632 the range in (A) absolute mMAXP, (B) absolute mMEANP, (C) relative mMAXP and (D)

633 relative mMEANP. at each subsample given by 1000 randomly generated subsamples at a

634 walking speed of $1.3 \mathrm{~m} / \mathrm{s}$. Data for individual subjects are shown as grey diamonds, with the

635 average for all subjects (blue diamonds and red solid line) and the subjects with the highest

636 (green diamonds and thin dashed red line) and lowest (red diamonds and thick dashed red line)

637 ranges highlighted. Dashed vertical lines on C and D indicate the $n$ steps at which percentage

638 values of the data mean reach specific thresholds (e.g. $5 \%, 10 \%$ ) of the value at the full sample

639 size.

640

641 Figure 4. The relationship between intra-subject sample size (number of steps per subject) and

642 (A) the minimum and maximum values recovered for mMAXP and (B) mMAXP in Subject 1

643 across a range of walking speeds $(1.1-1.9 \mathrm{~m} / \mathrm{s})$. These plots demonstrate that nly at sample sizes 
644 of 200 or more steps were the qualitative difference between speeds (i.e. higher mMEANP and

645 mMAXP values at higher walking speeds) preserved. At 200 or fewer steps for mMAXP and 50

646 or fewer steps for mMEANP, the range in values produced at adjacent walking speeds began to

647 overlap, raising the possibility of comparative analyses failing to correctly identify the

648 qualitative difference between walking speeds in this subject. Plots of (C) mMAXP and (D)

649 mMAXP for different subjects can be used to assess the predictive capability of different sample

650 sizes to correctly recover inter-subject differences in pressure at a single speed $(1.3 \mathrm{~m} / \mathrm{s})$. Similar

651 analyses can be used to assess the predictive capability of different sample and in (C) mean

652 maximum pressure and (D) and MMP for five exemplar subjects at $1.3 \mathrm{~m} / \mathrm{s}$. For (C) mMAXP, the

653 correct qualitative differences between subjects were preserved until the number of steps was

654 reduced to 25 or less, whereas for (D) mMEANP, the situation is more complex, but most

655 subjects begin to show overlapping ranges at steps of 200 or less, with high levels of overlap

656 (and therefore potential for incorrect identification of relative pressures) at 25 or fewer steps.

657

658 Figure 5. The relationship between intra-subject sample size (number of steps per subject) and

659 the range in absolute mean values for centroid pixel in the (A) heel, (B) midfoot, (C) lateral

660 (MT5) and (D) medial (MT1) forefoot, and (E) hallux in all subjects at $1.3 \mathrm{~m} / \mathrm{s}$.

661

662 Figure 6. The relationship between intra-subject sample size (number of steps per subject) and

663 the range in relative mean value for centroid pixel in the (A) heel, (B) midfoot, (C) lateral (MT5)

664 and (D) medial (MT1) forefoot, and (E) hallux in all subjects at $1.3 \mathrm{~m} / \mathrm{s}$.

665

Peer] reviewing PDF | (2020:12:55991:1:0:NEW 13 May 2021) 
666 Figure 7. (A) The relationship between intra-subject sample size (number of steps per subject)

667 and the range in mean MSE at each subsample given by 1000 randomly generated subsamples at 668 a walking speed of $1.3 \mathrm{~m} / \mathrm{s}$. This relationship is well described by exponential curves in all 669 subjects, which are plotted here for the overall mean of all subjects and the subjects with the 670 highest (7) and lowest (13) overall MSE. (B) The relationship between intra-subject sample size 671 (number of steps per subject) and the normalized range in mean MSE at each subsample. The 672 normalized range in MSE is plotted as a percentage of mean MSE of the full dataset for each 673 subject walking at $1.3 \mathrm{~m} / \mathrm{s}$. When the sample size is $n=>400$ steps, then the range in mean MSE 674 is less than 5\% higher than the full dataset values for each subject. The observed range in mean $675 \mathrm{MSE}$ at smaller dataset sizes $(n=25$ steps) is more than $50 \%$ higher than the total dataset values 676 observed for each subject.

677

678 Figure 8. Mean peak plantar images (left, large images) and the peak plantar pressure records 679 from the first 12 consecutive steps (right, smaller images) from the subjects with the highest (A, 680 subject 7) and lowest (B, subject 13) MSE to illustrate high levels of step-to-step variability in 681 pressure distribution at $1.3 \mathrm{~m} / \mathrm{s}$. 


\section{Figure 1}

Overview of the plantar pressure metrics used in the intra-subject (number of steps) subsampling analysis.

We investigated the sensitivity of three different metric types to the number of steps analysed within each subject. These were $(A)$ the simple 'whole-foot' mean (mMEANP) and maximum pressure (mMAXP), and (B) the absolute values of pixels representing the approximate centroid of the heel, midfoot, lateral and medial forefoot (MT5 and MT1 above) and the hallux. (C) To examine how the number of steps analysed within a subject may influence the foot as a whole (i.e. the sum of variability across all pixels in each record), and therefore sample size implications for pixel-level vector analyses of pressure we calculated the global mean square error (MSE) of all pixels in each pressure record following McClymont et al., (2016). 
A

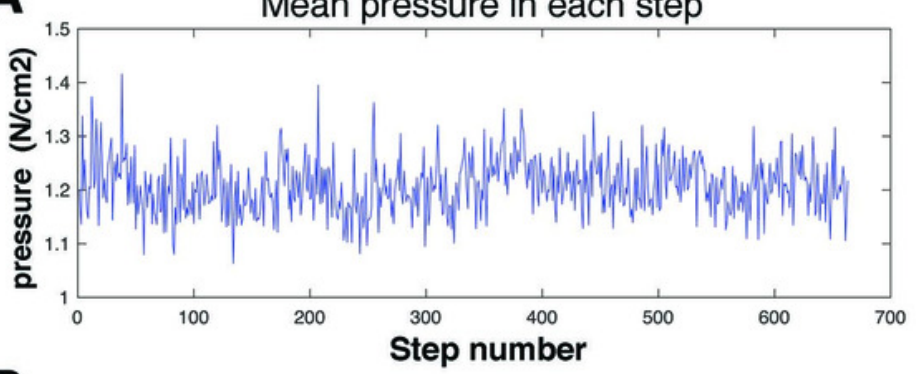

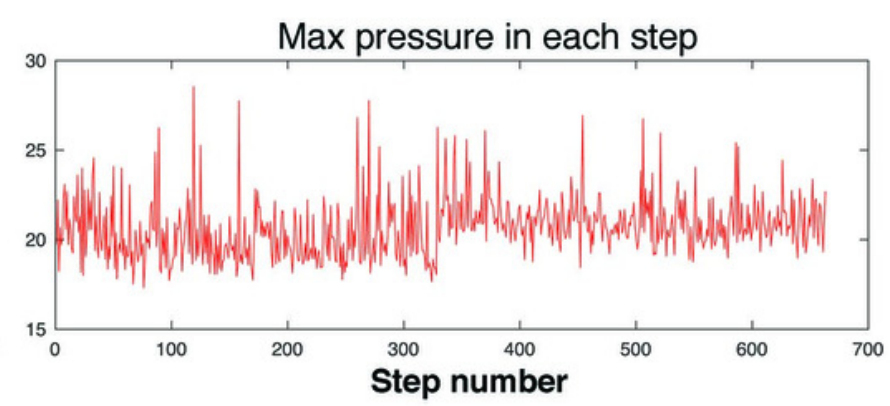

B
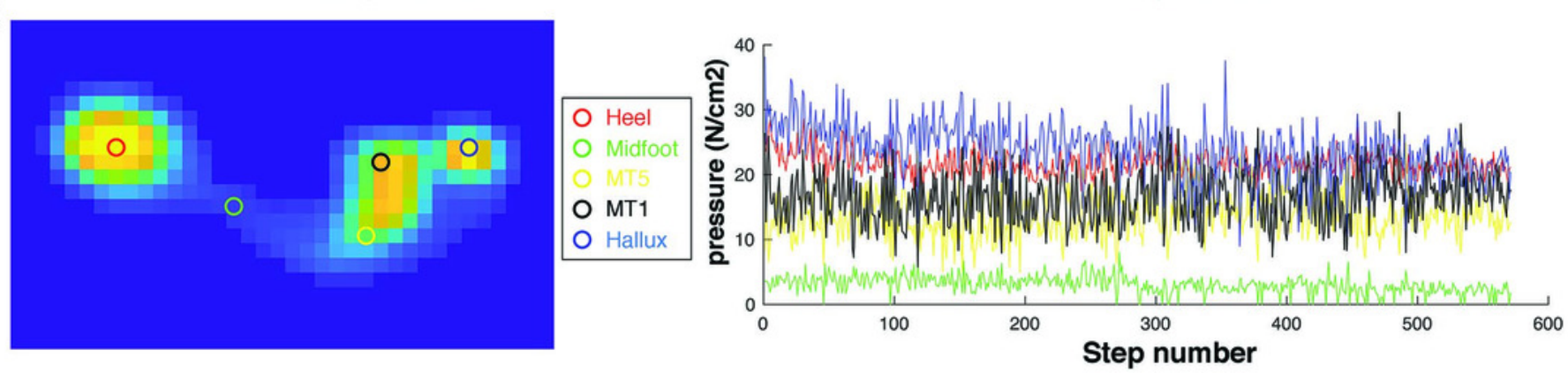

C
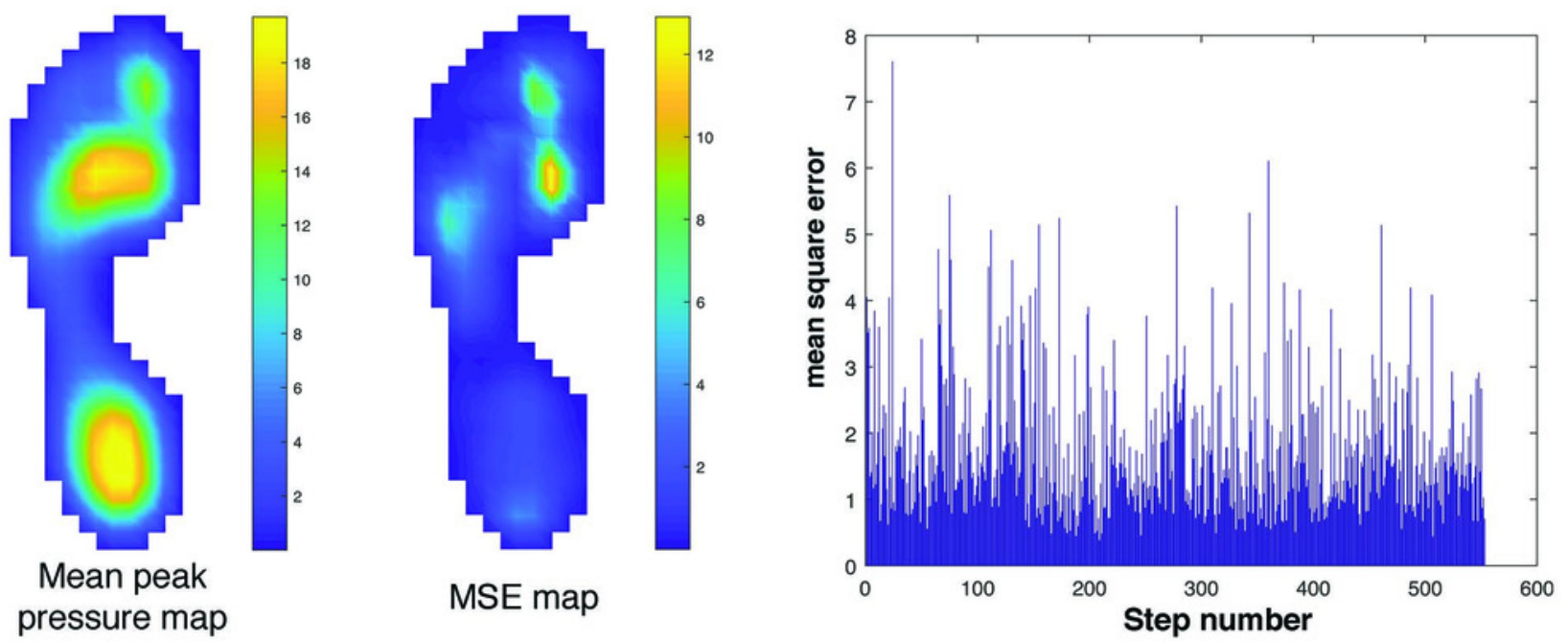


\section{Figure 2}

Conceptual example of the subsampling analysis applied to the various pressure metrics shown in Figure 1.

(A) 1000 random subsamples of the pressure metrics were extracted from each subject's overall total number of steps at subsamples of 5, 25, 50, 100, 200, 300, 400 and 500 steps (represented as blue diamond data points in A). As the number of steps included in any one of 1000 subsampled populations decreases it would be expected that the range in the absolute value of the metric pressure metric would increase, reflecting (for example) the relatively greater impact of 'extreme' high and low values from specific steps. In other words, the absolute range of values for the pressure metric (dashed blue lines delimiting the maximum - minimum values from the 1000 subsampled populations) would be expected to increase as the number of steps included decreases, as shown in (B). The absolute and relative magnitude of metric ranges are plotted against sample size ( $n$ steps) in subsequent plots to make comparisons across subjects and speeds, as shown in (B). 

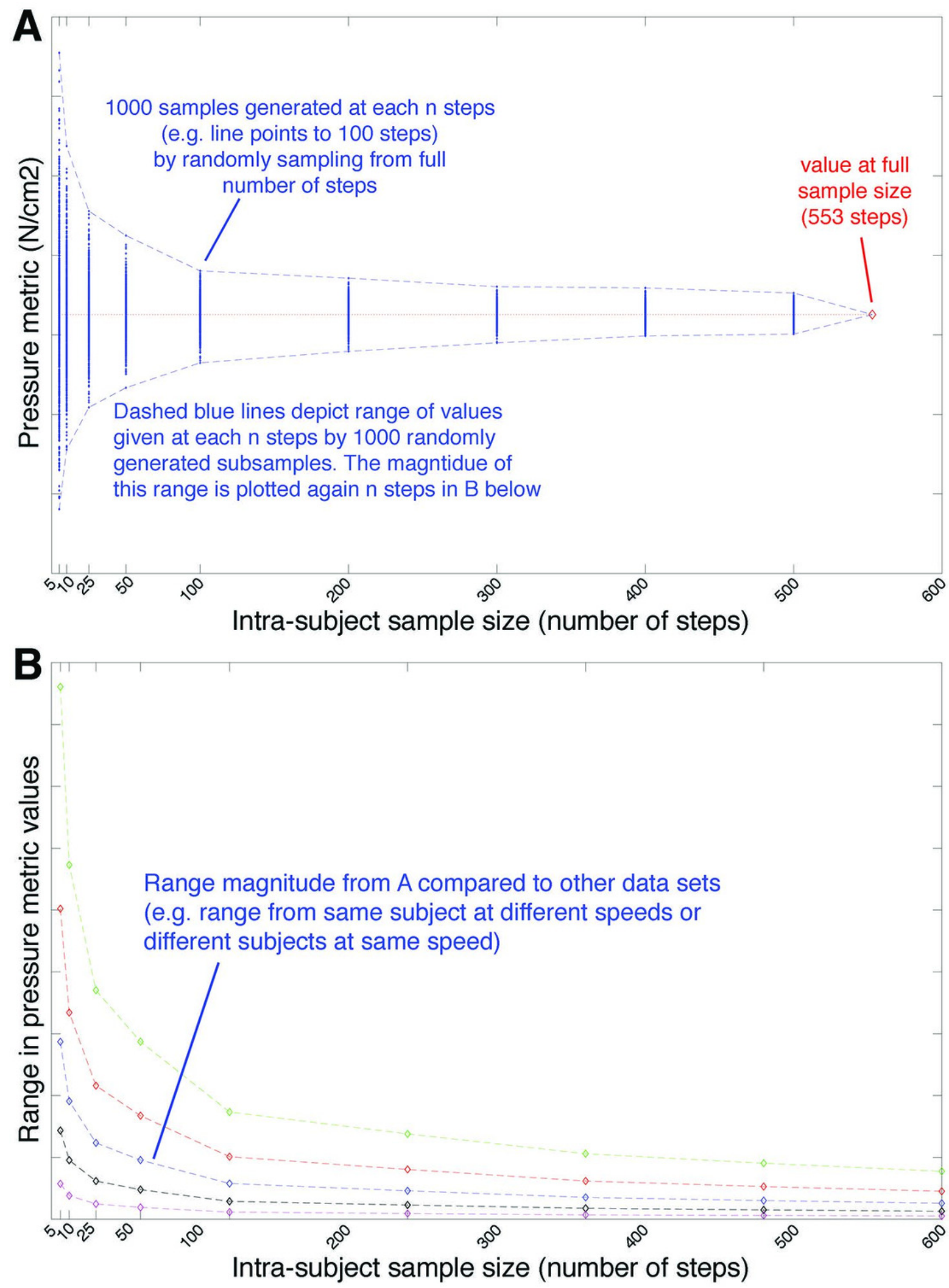


\section{Figure 3}

The relationship between intra-subject sample size (number of steps per subject) and the range of values for simple pressure metrics.

The relationship between intra-subject sample size (number of steps per subject) and the range in (A) absolute mMAXP, (B) absolute mMEANP, (C) relative mMAXP and (D) relative mMEANP. at each subsample given by 1000 randomly generated subsamples at a walking speed of $1.3 \mathrm{~m} / \mathrm{s}$. Data for individual subjects are shown as grey diamonds, with the average for all subjects (blue diamonds and red solid line) and the subjects with the highest (green diamonds and thin dashed red line) and lowest (red diamonds and thick dashed red line) ranges highlighted. Dashed vertical lines on $C$ and $D$ indicate the $n$ steps at which percentage values of the data mean reach specific thresholds (e.g. $5 \%, 10 \%$ ) of the value at the full sample size. 

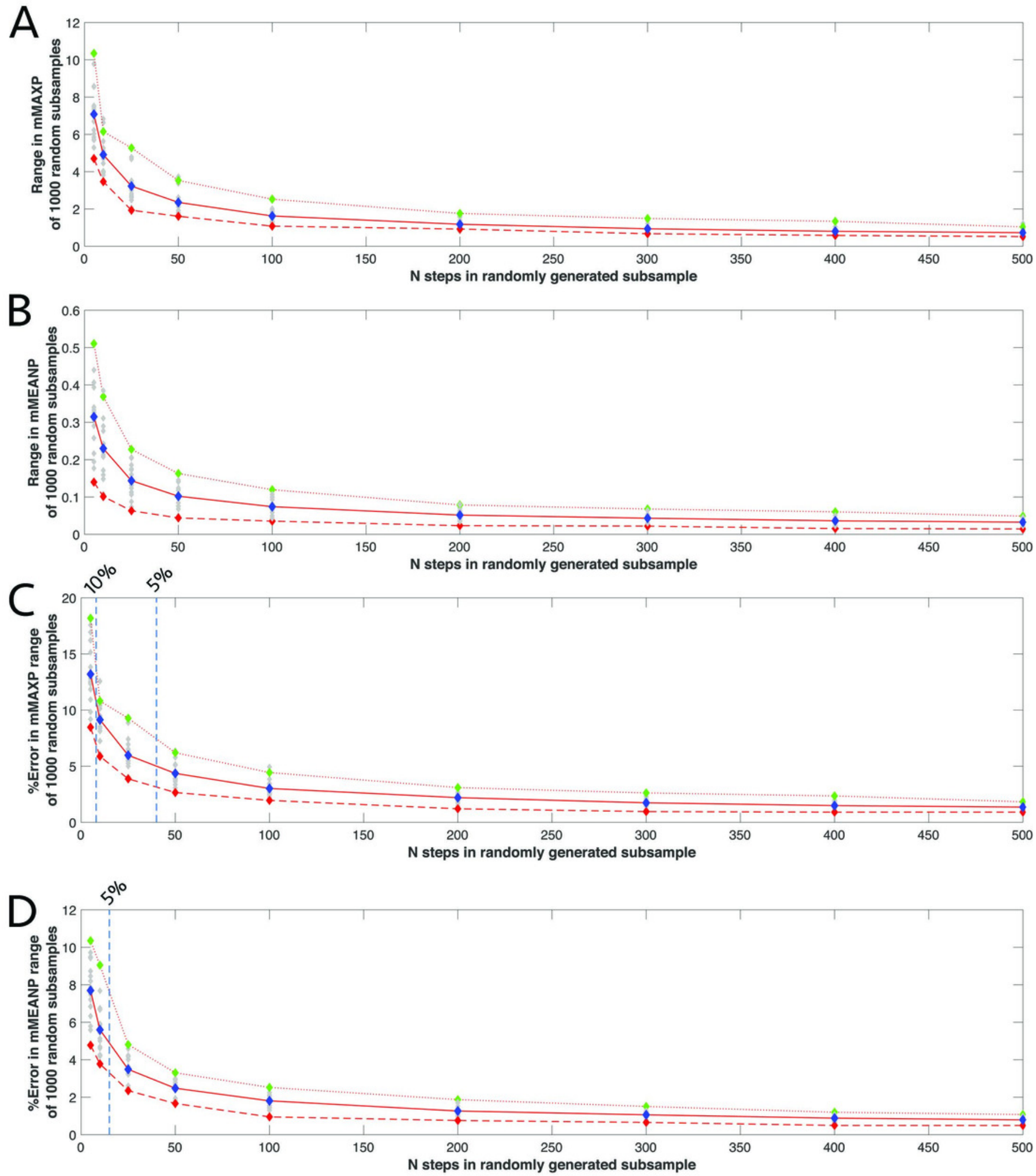

Individual subject range value 


\section{Figure 4}

Intra-subject sample size effects on the patterns recovered when assessing changes in pressure with speed within subjects, and in relative pressures across subjects.

The relationship between intra-subject sample size (number of steps per subject) and (A) the minimum and maximum values recovered for mMAXP and (B) mMAXP in Subject 1 across a range of walking speeds (1.1-1.9m/s). These plots demonstrate that nly at sample sizes of 200 or more steps were the qualitative difference between speeds (i.e. higher mMEANP and mMAXP values at higher walking speeds) preserved. At 200 or fewer steps for mMAXP and 50 or fewer steps for mMEANP, the range in values produced at adjacent walking speeds began to overlap, raising the possibility of comparative analyses failing to correctly identify the qualitative difference between walking speeds in this subject. Plots of (C) MMAXP and (D) mMAXP for different subjects can be used to assess the predictive capability of different sample sizes to correctly recover inter-subject differences in pressure at a single speed $(1.3 \mathrm{~m} / \mathrm{s})$. Similar analyses can be used to assess the predictive capability of different sample and in (C) mean maximum pressure and (D) and MMP for five exemplar subjects at $1.3 \mathrm{~m} / \mathrm{s}$. For (C) mMAXP, the correct qualitative differences between subjects were preserved until the number of steps was reduced to 25 or less, whereas for (D) mMEANP, the situation is more complex, but most subjects begin to show overlapping ranges at steps of 200 or less, with high levels of overlap (and therefore potential for incorrect identification of relative pressures) at 25 or fewer steps. 

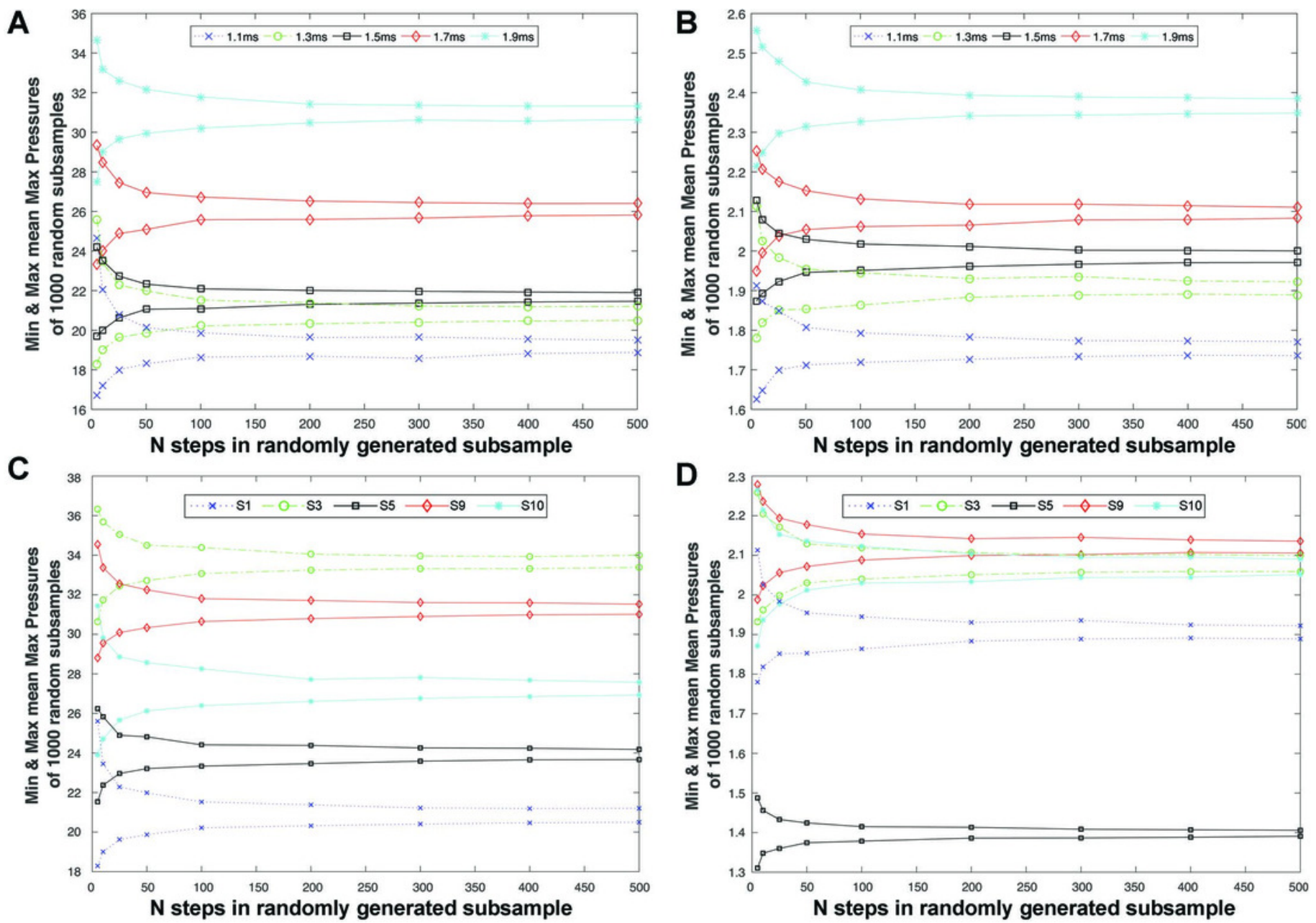


\section{Figure 5}

The relationship between intra-subject sample size (number of steps per subject) and the range in absolute pressure values for different foot regions.

The relationship between intra-subject sample size (number of steps per subject) and the range in absolute mean values for centroid pixel in the (A) heel, (B) midfoot, (C) lateral (MT5) and (D) medial (MT1) forefoot, and (E) hallux in all subjects at $1.3 \mathrm{~m} / \mathrm{s}$. 

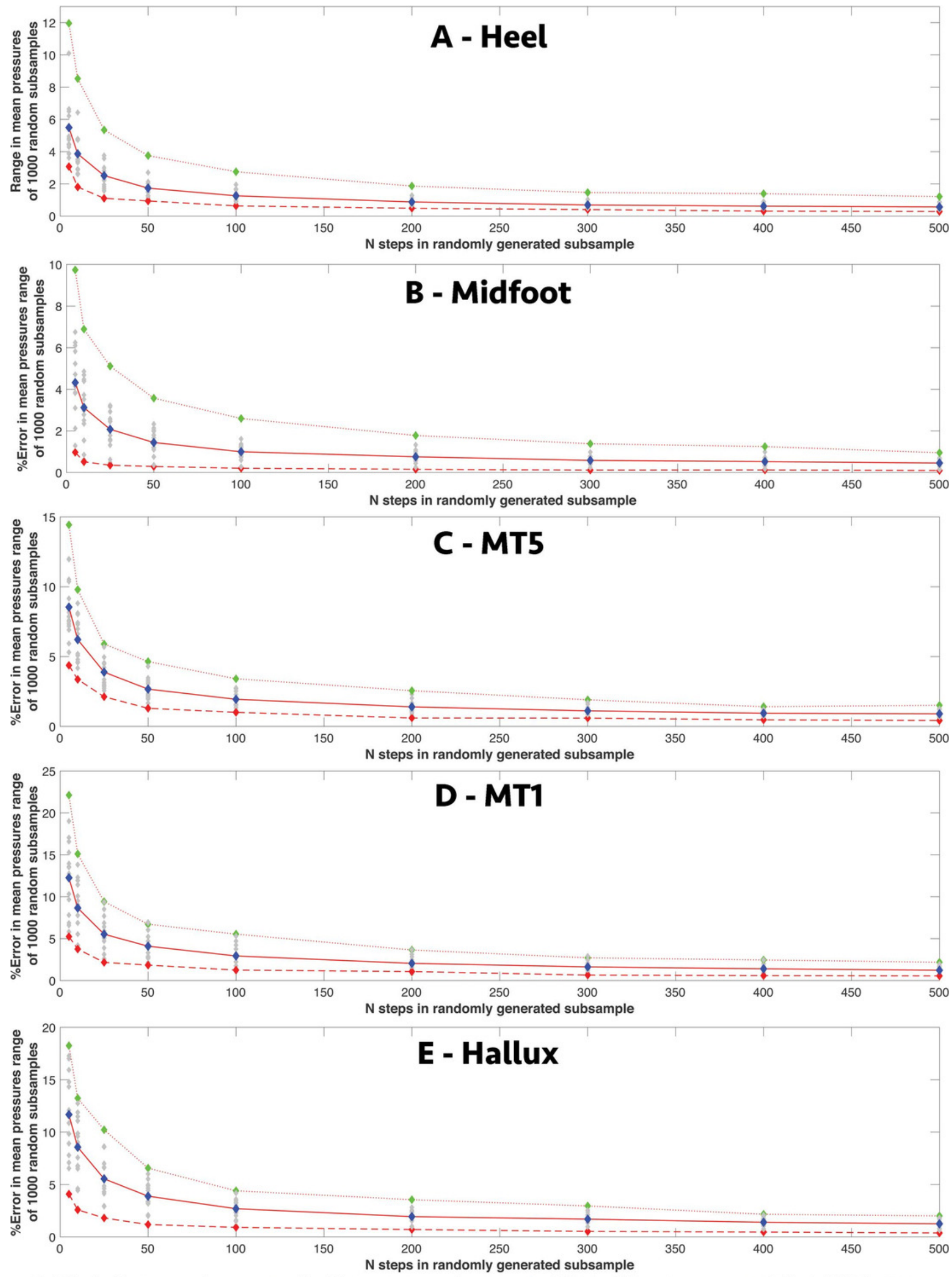

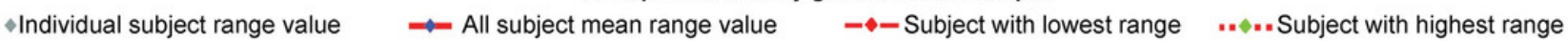




\section{Figure 6}

The relationship between intra-subject sample size (number of steps per subject) and the range in relative pressure values for different foot regions.

The relationship between intra-subject sample size (number of steps per subject) and the range in relative mean value for centroid pixel in the (A) heel, (B) midfoot, (C) lateral (MT5) and (D) medial (MT1) forefoot, and (E) hallux in all subjects at $1.3 \mathrm{~m} / \mathrm{s}$. 

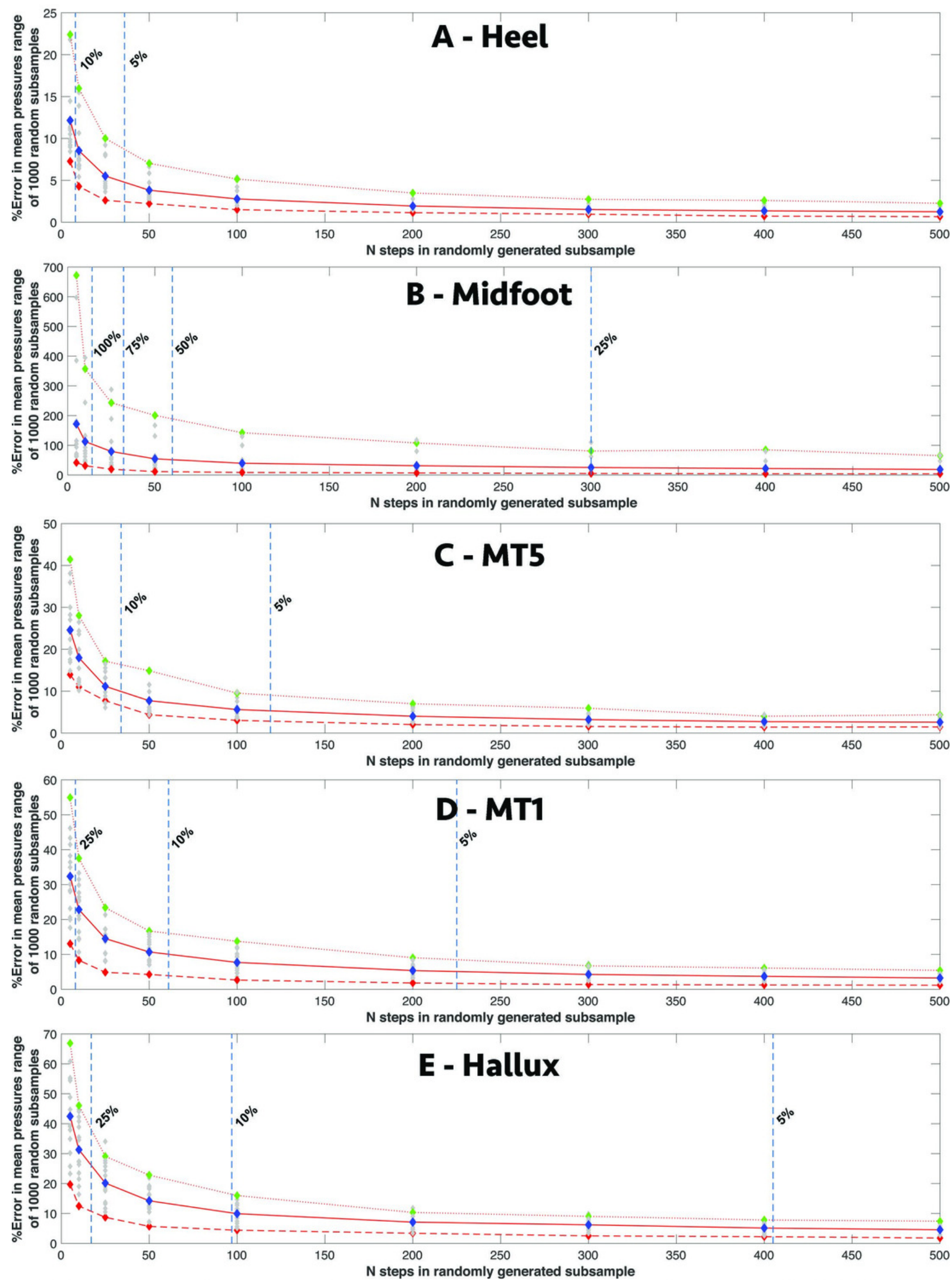

-Individual subject range value $\quad \rightarrow$ All subject mean range value $\quad-\$-$ Subject with lowest range $\quad \ldots \bullet$...Subject with highest range 


\section{Figure 7}

The relationship between intra-subject sample size (number of steps per subject) and the range in 'whole-foot' plantar pressure mean MSE.

(A) The relationship between intra-subject sample size (number of steps per subject) and the range in mean MSE at each subsample given by 1000 randomly generated subsamples at a walking speed of $1.3 \mathrm{~m} / \mathrm{s}$. This relationship is well described by exponential curves in all subjects, which are plotted here for the overall mean of all subjects and the subjects with the highest (7) and lowest (13) overall MSE. (B) The relationship between intra-subject sample size (number of steps per subject) and the normalized range in mean MSE at each subsample. The normalized range in MSE is plotted as a percentage of mean MSE of the full dataset for each subject walking at $1.3 \mathrm{~m} / \mathrm{s}$. When the sample size is $n=>400$ steps, then the range in mean MSE is less than $5 \%$ higher than the full dataset values for each subject. The observed range in mean MSE at smaller dataset sizes ( $n=25$ steps) is more than $50 \%$ higher than the total dataset values observed for each subject. 


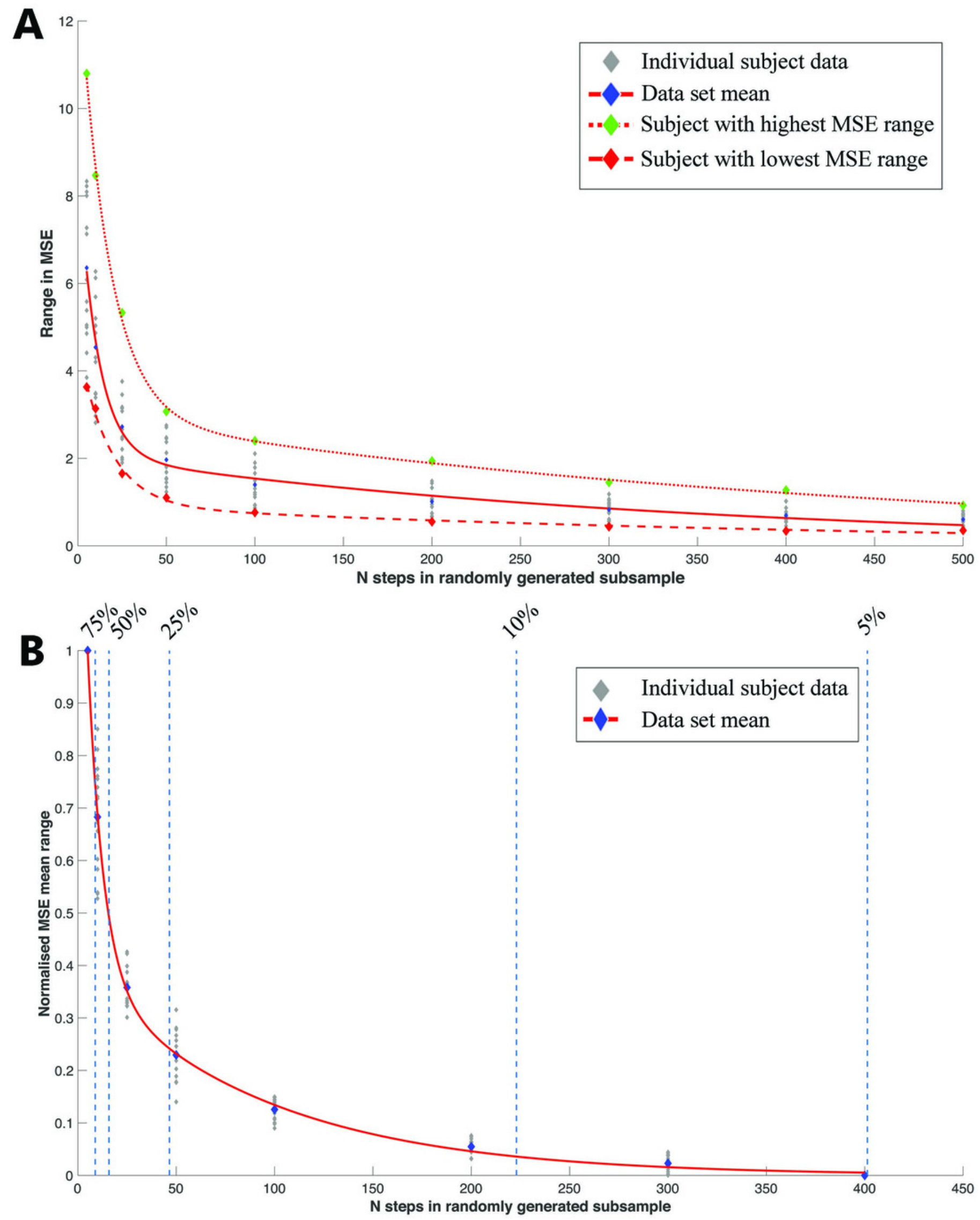




\section{Figure 8}

Examples of intra-subject (step-to-step) variation in peak pressure magntidue and distribution.

Mean peak plantar images (left, large images) and the peak plantar pressure records from the first 12 consecutive steps (right, smaller images) from the subjects with the highest ( $A$, subject 7) and lowest (B, subject 13) MSE to illustrate high levels of step-to-step variability in pressure distribution at $1.3 \mathrm{~m} / \mathrm{s}$.
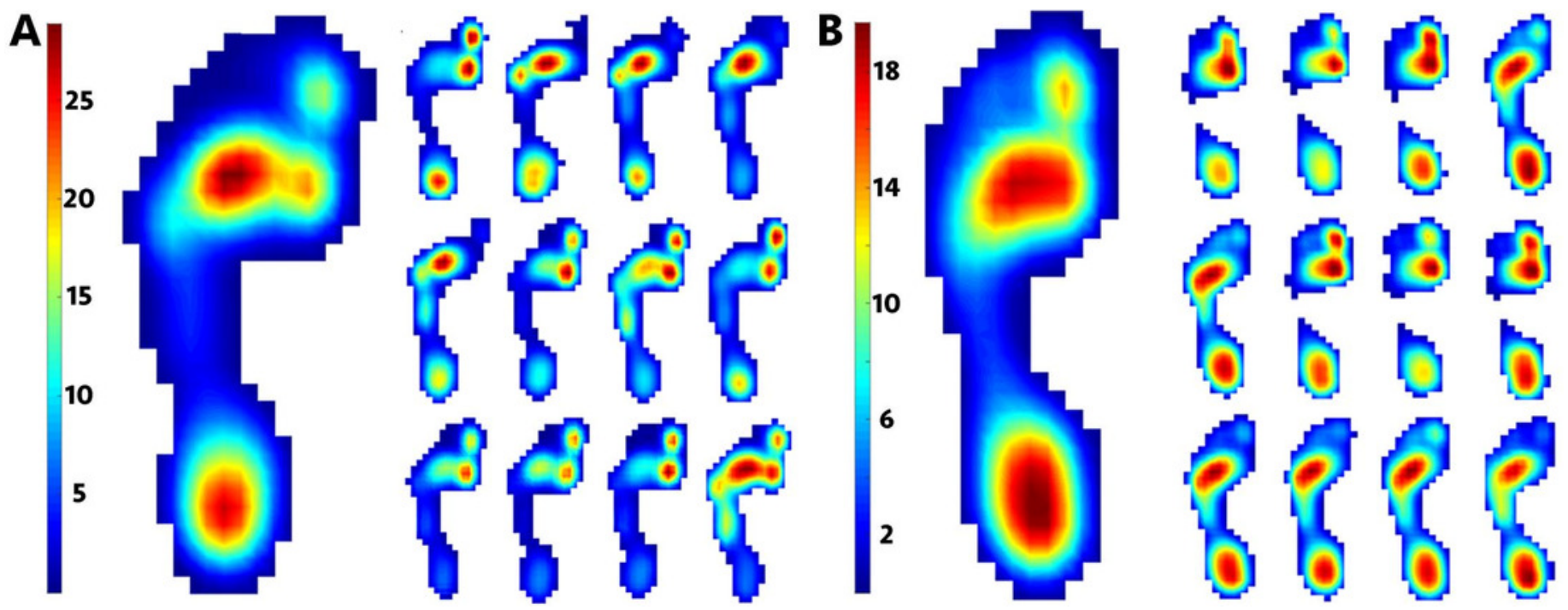